\title{
Characterization of InGaN/GaN quantum well growth using monochromated valence electron energy loss spectroscopy
}

\author{
Justinas Palisaitis, Anders Lundskog, Urban Forsberg, Erik Janzén, Jens Birch, \\ Lars Hultman, Pe and Persson
}

\section{Linköping University Post Print}

N.B.: When citing this work, cite the original article.

Original Publication:

Justinas Palisaitis, Anders Lundskog, Urban Forsberg, Erik Janzén, Jens Birch, Lars Hultman, Pe and Persson, Characterization of InGaN/GaN quantum well growth using monochromated valence electron energy loss spectroscopy, 2014, Journal of Applied Physics, (115), 034302. http://dx.doi.org/10.1063/1.4861179

Copyright: American Institute of Physics (AIP) http://www.aip.org/

Postprint available at: Linköping University Electronic Press http://urn.kb.se/resolve?urn=urn:nbn:se:liu:diva-85903 


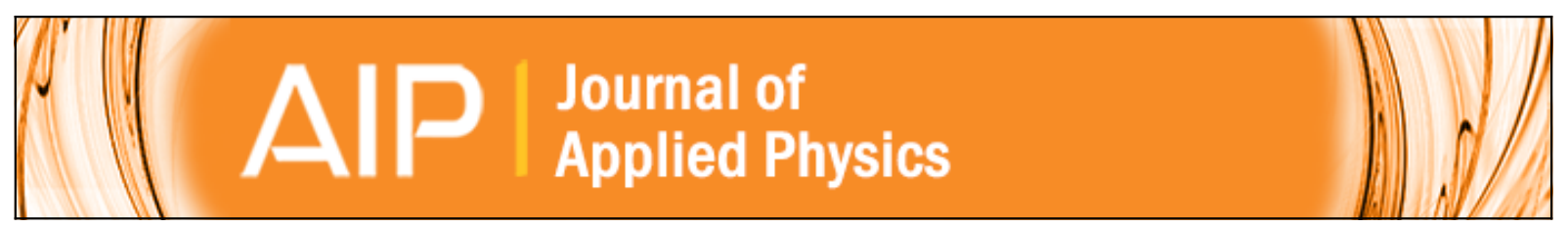

Characterization of InGaN/GaN quantum well growth using monochromated valence electron energy loss spectroscopy

J. Palisaitis, A. Lundskog, U. Forsberg, E. Janzén, J. Birch, L. Hultman, and P. O. Å. Persson

Citation: Journal of Applied Physics 115, 034302 (2014); doi: 10.1063/1.4861179

View online: http://dx.doi.org/10.1063/1.4861179

View Table of Contents: http://scitation.aip.org/content/aip/journal/jap/115/3?ver=pdfcov

Published by the AIP Publishing

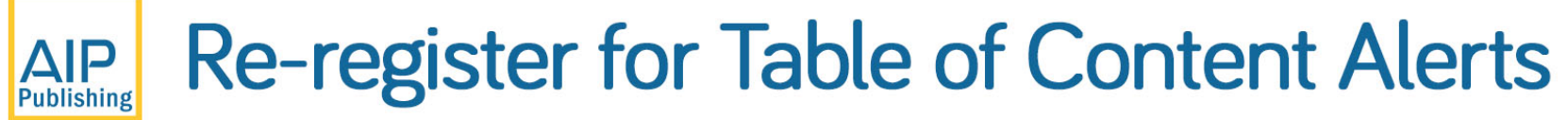




\title{
Characterization of InGaN/GaN quantum well growth using monochromated valence electron energy loss spectroscopy
}

\author{
J. Palisaitis, ${ }^{1, a)}$ A. Lundskog, ${ }^{2}$ U. Forsberg, ${ }^{2}$ E. Janzén, ${ }^{2}$ J. Birch, ${ }^{1}$ L. Hultman, ${ }^{1}$ \\ and P. O. A. Persson ${ }^{1}$ \\ ${ }^{1}$ Thin Film Physics Division, Department of Physics, Chemistry and Biology (IFM), Linköping University, \\ SE-581 83 Linköping, Sweden \\ ${ }^{2}$ Semiconductor Materials Division, Department of Physics, Chemistry and Biology (IFM), Linköping \\ University, SE-581 83 Linköping, Sweden
}

(Received 29 October 2013; accepted 18 December 2013; published online 16 January 2014)

\begin{abstract}
The early stages of InGaN/GaN quantum well growth for In-reduced conditions have been investigated for varying thickness and composition of the wells. The structures were studied by monochromated scanning transmission electron microscopy-valence electron energy loss spectroscopy spectrum imaging at high spatial resolution. It is found that beyond a critical well thickness and composition, quantum dots (width $>20 \mathrm{~nm}$ ) are formed inside the well. These are buried by compositionally graded InGaN, which is formed as $\mathrm{GaN}$ is grown while residual $\mathrm{In}$ is incorporated into the growing structure. It is proposed that these dots act as carrier localization centers inside the quantum wells. (C) 2014 AIP Publishing LLC. [http://dx.doi.org/10.1063/1.4861179]
\end{abstract}

\section{INTRODUCTION}

III-Nitrides receive scientific and industrial attention due to their unique fundamental properties. The technology based on $\mathrm{AlN}, \mathrm{GaN}$, and $\mathrm{InN}$ as well as their ternary and quaternary compounds has been successfully implemented in a wide range of applications in both optical and electronic devices, which are operated at high temperatures, high frequencies, and high power. ${ }^{1-4}$ GaN-based heterostructures like InGaN/GaN single or multiple quantum wells (QWs) dimensioned on the nanoscale are employed as the active layer in high-brightness light emitting diodes, laser diodes, and multi-junction solar cells with high internal quantum efficiencies. ${ }^{5,6}$ The direct bandgap of InGaN allows for tunable color emission, covering the whole visible and parts of the ultraviolet and infrared spectral ranges. Fabrication of high-quality structures throughout the compositional range is challenging, primarily due to the onset of phase separation by spinodal decomposition. This comes as a consequence of the large miscibility gap as originally investigated by Ho and Stringfellow. ${ }^{7}$ Later, Karpov ${ }^{8}$ outlined a pathway for reducing the miscibility gap significantly, by crystal growth at higher temperatures and at high strain. Multiple electron microscopy studies have been conducted on this topic, revealing nanoscale $(\sim 2-5 \mathrm{~nm})$ clusters of high In content, which are recognized as "dots". $9-11$ While the majority of these studies are focused on structures, which are grown at conditions and In concentrations where spinodal decomposition may occur, there is a lack of investigations on the early stages of nucleation of a QW for compositions outside the miscibility gap.

In this paper, we present an investigation by monochromated scanning transmission electron microscopy (STEM), employing mapping by valence electron energy loss spectroscopy (VEELS) of InGaN/GaN QW structures, designed for different thicknesses and compositions. The investigation reveals a pseudomorphic growth of the wells wherein

\footnotetext{
a) Author to whom correspondence should be addressed. Electronic mail: juspa@ifm.liu.se
}

pronounced quantum dot (QD) like features emerge as arranged compositional variations inside the QW upon a critical thickness and composition. It is proposed that this occurs as a consequence of Stranski-Krastanov-type growth. The QDs are laterally and vertically overgrown by In-reduced InGaN where the In is incorporated from residual In segregating on the advancing surface.

\section{EXPERIMENTAL DETAILS}

Two heterostructure samples containing InGaN/GaN MQWs were investigated in this study. The samples were grown in a low pressure hot-wall metal organic chemical vapour deposition (MOCVD) reactor. Trimethylaluminum (TMAl), trimethylgallium (TMGa), trimethylindium (TMIn), and ammonia $\left(\mathrm{NH}_{3}\right)$ were used as precursors for $\mathrm{Al}, \mathrm{Ga}, \mathrm{In}$, and $\mathrm{N}$, respectively, with nitrogen as carrier gas. QW structures were deposited on top of a $\sim 2 \mu \mathrm{m}$ thick, low defect-density GaN(0001) buffer layer. The GaN buffer was grown on top of a $\sim 140 \mathrm{~nm}$ thick $\mathrm{AlN}(0001)$ nucleation layer and nominally on-axis c-plane $4 \mathrm{H}-\mathrm{SiC}(0001)$ substrate. The first InGaN/GaN sample was grown at $800{ }^{\circ} \mathrm{C}$ and hosts six periods of low-In content QWs with increasing QW thickness towards the surface, where each QW is separated by a GaN interlayer of constant thickness. Low-In content QW sample is referred as the "L-In." The second sample was grown using the same sequence as for the first, except at a temperature of $760^{\circ} \mathrm{C}$ and with an increased TMIn flow resulting in six high-In content QWs, refereed as "H-In." The In content in the grown QWs was tuned by applying different TMIn flow rates, $25 \mathrm{ml} / \mathrm{min}$ for L-In and $75 \mathrm{ml} / \mathrm{min}$ for H-In, respectively, and the thickness of the different QWs was obtained by adjusting the growth time. A detailed description of the growth conditions used for similar structures can be found elsewhere. ${ }^{12,13}$

Electron-transparent cross-sectional specimens were prepared from the as-grown material using the traditional "sandwich" approach. First the samples were cut, mounted 
in a titanium grind and glued, followed by mechanical polishing to $\sim 50 \mu \mathrm{m}$ thickness. $\mathrm{Ar}^{+}$ion milling at $5 \mathrm{keV}$ and $5^{\circ}$ from both sides was performed in a Gatan precision ion polishing system (PIPS). The $\mathrm{Ar}^{+}$ion energy was gradually reduced to $2 \mathrm{keV}$ during the final step of milling to minimize the surface damage on the samples.

STEM combined with high angle annular dark field imaging (HAADF) and STEM VEELS analyses were performed in the double-corrected Linköping FEI Titan ${ }^{3}$ 60-300, operated at $300 \mathrm{kV}$. The microscope exhibits a high brightness field emission gun (XFEG) and monochromator to filter the energy spread of the electron beam for high energy resolution spectroscopy. EELS analyses were performed using a Gatan GIF Quantum ERS post-column imaging filter with $1 \mathrm{kHz}$ spectral acquisition. ${ }^{14}$

High resolution STEM (HRSTEM) imaging was recorded by using an optimized $30 \mathrm{mrad}$ convergence angle, which provided sub-angstrom resolution probes and 70-140 mrad HAADF detector acceptance angle range. Spectroscopy was performed by using an energy spread of the primary electron beam of $0.2 \mathrm{eV}$, optimized for current, as defined by the full width at half maximum (FWHM) of the zero loss peak, throughout the VEELS measurements. The convergence semi-angle in monochromated mode was set to $20 \mathrm{mrad}$, providing a sub-Ångstrom probe with $0.3 \mathrm{nA}$ of current. VEELS spectrum images of $250 \times 250 \mathrm{px}^{2}$ were recorded using a $0.025 \mathrm{eV} / \mathrm{channel}$ energy dispersion, a collection semi-angle of $6 \mathrm{mrad}, 5 \mathrm{~ms}$ dwell time for each pixel, and a total of $7 \mathrm{~min}$ for recording the complete spectrum image. The overview VEELS spectrum images were recorded with a pixel width of $1.25 \mathrm{~nm} / \mathrm{px}$, while the high resolution spectrum images were recorded with $0.16 \mathrm{~nm} / \mathrm{px}$. The spectrum images were also recorded for a higher signalto-noise ratio according to the method proposed by Bosman and Keast. ${ }^{15}$ The peak energy position of the bulk plasmon, $\mathrm{E}_{\mathrm{p}}$, was mapped across the structures and obtained by an initial zero loss peak fitting and re-alignment of the spectrum image for energy drift. This was followed by Fourier-log deconvolution for plural scattering removal. Finally, applying a single $2 \mathrm{eV}$ FWHM Gaussian to the low-loss spectrum, the EELS spectrum was fitted by a nonlinear least-squares (NLLS) curve-fitting method centered around the most intense part of the plasmon peak for extracting $E_{p}$ with a fitting accuracy of $0.01 \mathrm{eV} .^{16,17}$

\section{RESULTS AND DISCUSSION}

Low magnification STEM overview images of the InGaN/GaN QW structures, of low and high In content in the InGaN wells, are shown in Figure 1(a) (L-In) and 1(b) (H-In), respectively. Both samples display the AlN nucleation and $\mathrm{GaN}$ buffer layers, which appear to be of similar thickness and quality. However, the top part of the grown samples, which hosts the InGaN/GaN QWs, differs structurally. As can be seen, the L-In sample exhibits a relatively flat surface (Figure 1(a)), with occasional V-shaped defects present in the structure. The formation mechanisms of such defects were extensively investigated in the past. ${ }^{18,19}$ In contrast, the H-In sample exhibits a considerably rough surface.

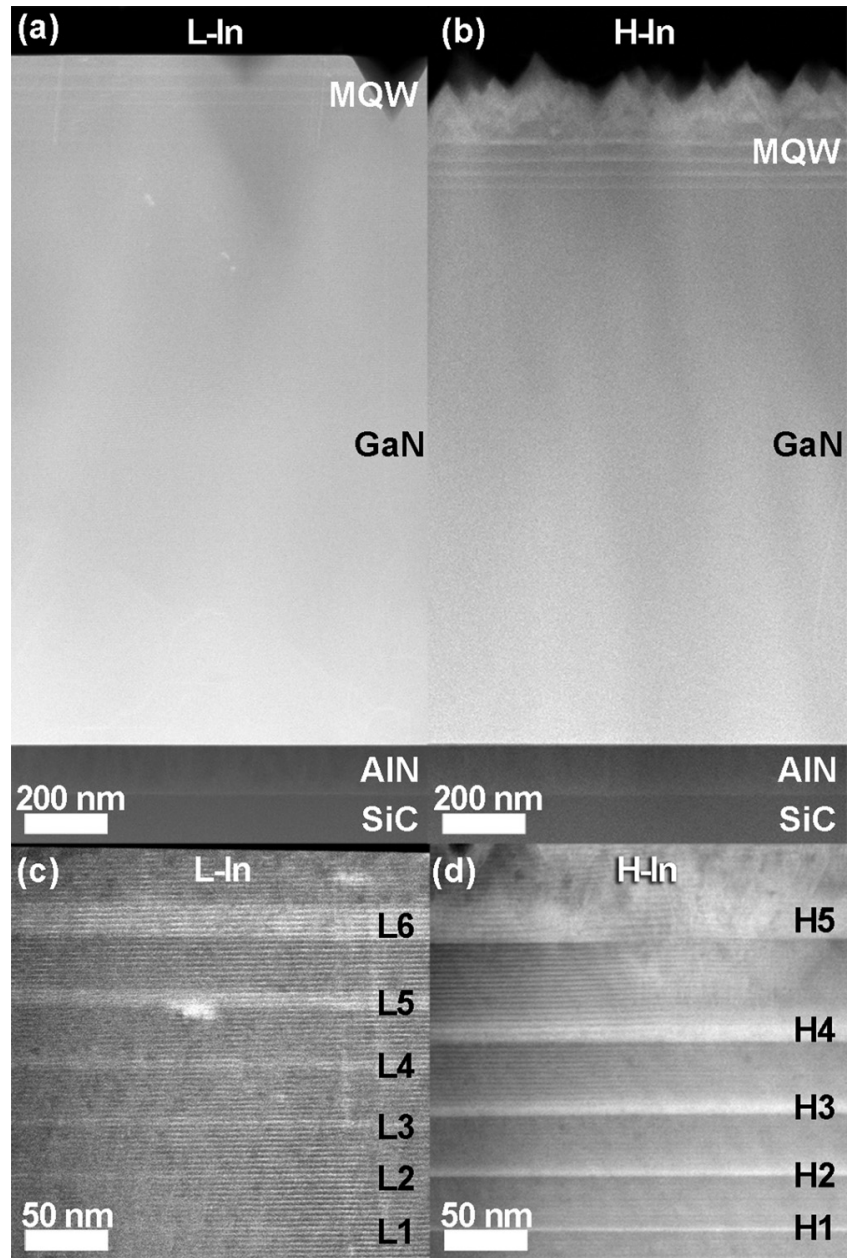

FIG. 1. Low magnification STEM images providing full overview of the MOCVD grown III-nitride heterostructure with (a) low In (L-In) and (b) high In (H-In) content QWs. Higher magnification STEM images showing the InGaN/GaN QW region with increasing QW thickness towards the surface for (c) L-In and (d) H-In content samples.

This surface roughness is proposed to occur as a consequence of plastic strain relaxation, which occurs when a high In content InGaN layer exceeds a critical thickness where the structure can no longer accommodate for the built-in strain. ${ }^{20}$ The strain relaxation results in a burst of structural defects such as misfit dislocations, stacking faults, domain boundaries, and V-shaped defects.

Corresponding higher magnification STEM images of the InGaN/GaN QW regions are shown in Figures 1(c) and 1(d), respectively. The L-In sample reveals the six InGaN QWs of increasing well thickness towards the surface, which are separated by $\sim 30 \mathrm{~nm} \mathrm{GaN}$ interlayers. The QWs in the L-In sample are indexed as L1-starting from the bottom (thinnest well) and ending with L6 near the surface (thickest well). Some carbon contamination (accidentally applied during microscopy) is visible in the middle of L5. The H-In sample contains four continuous InGaN QWs with increasing well thickness from bottom to the top (H1-H4). A fifth layer, H5, can also be identified, although this is not continuous due to layer interruption by the rough surface. The well is locally considered in the following analyses. The wells are separated by $\sim 35 \mathrm{~nm} \mathrm{GaN}$ interlayers. 


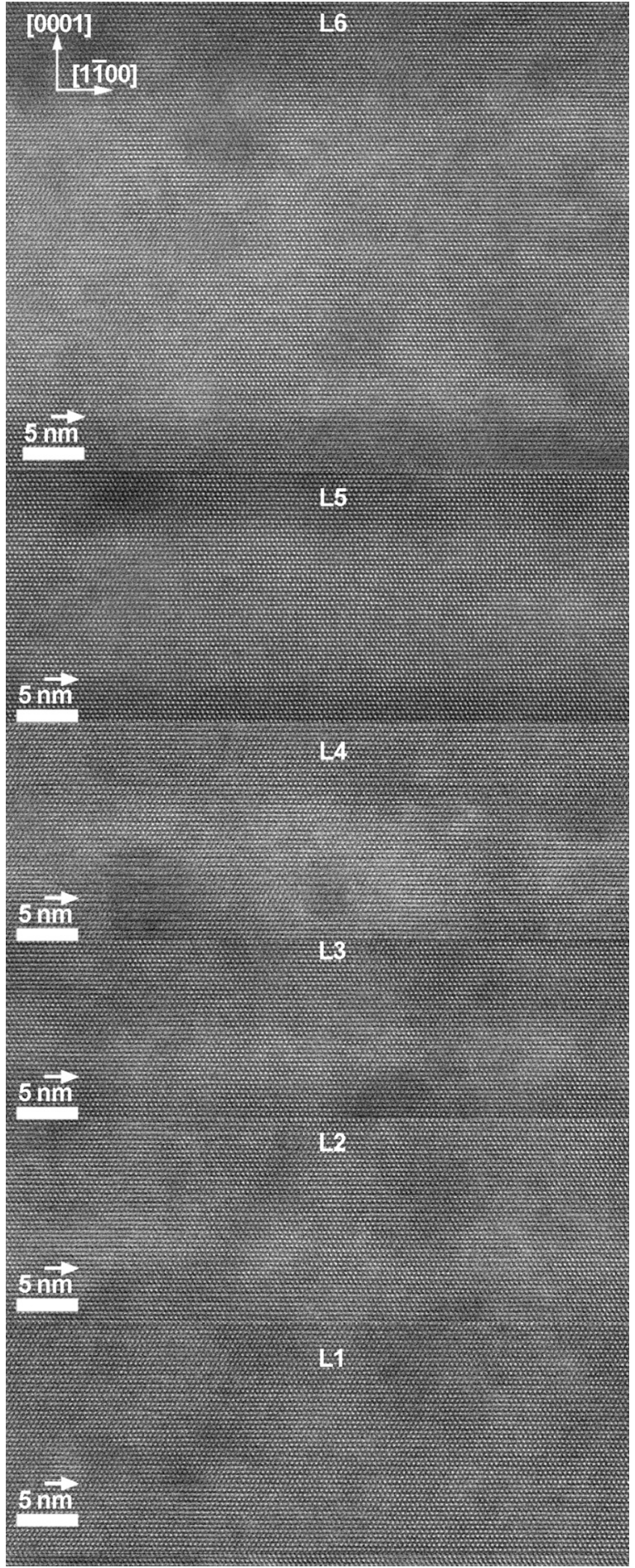

FIG. 2. Series of atomically resolved HRSTEM images showing each QW separately from the L-In content sample. The images are aligned with respect to the bottom of the QW interfaces, as indicated by an arrow.

HRSTEM imaging was further used to investigate the quantum wells, and the results are shown in Figures 2 and 3 where each well is individually imaged along the $[11 \overline{2} 0]$ zone axis. The atomically resolved HRSTEM images were obtained by employing strong $\mathrm{Z}$ contrast, thus the In containing $\mathrm{Ga}$ atomic columns produce more contrast in the QWs, compared to the interlayer columns. For the L-In sample, the Z-contrast caused by the low amount of incorporated In is not as strong as for the H-In sample, though the QWs are

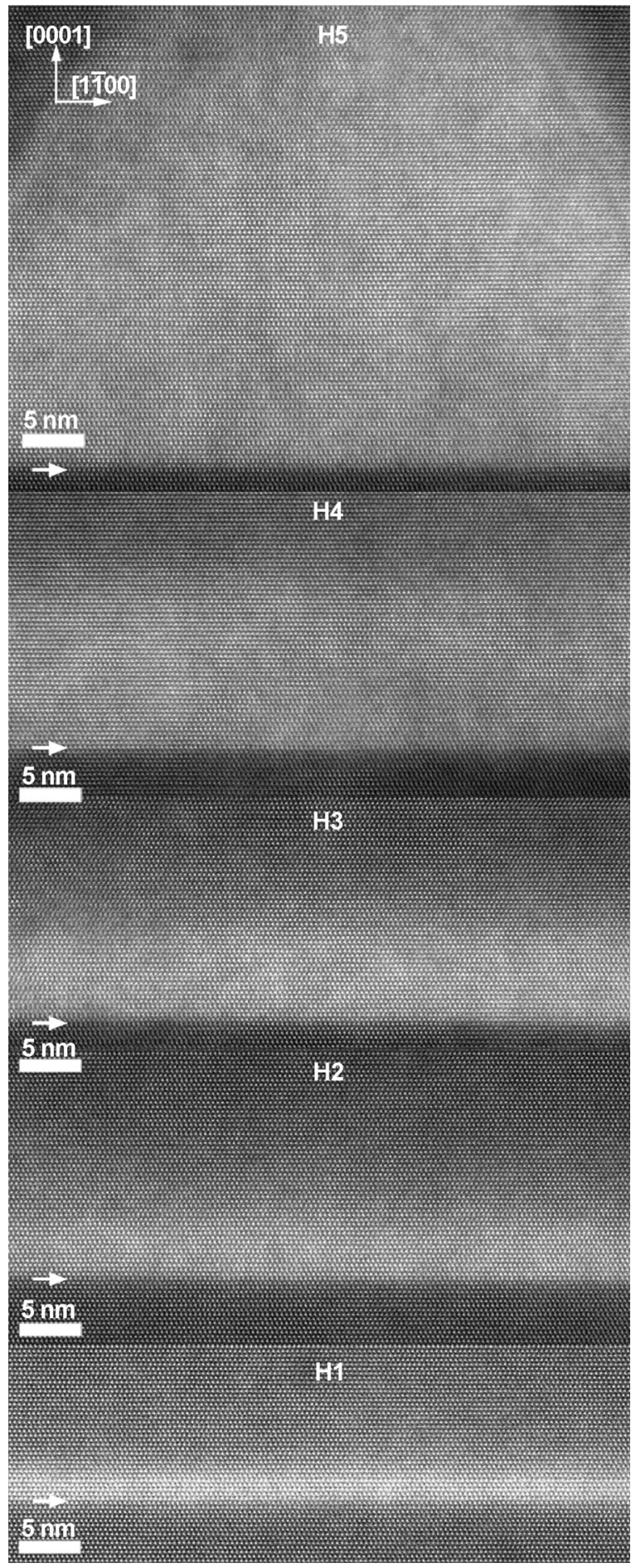

FIG. 3. Series of atomically resolved HRSTEM images showing each QW separately from the H-In content sample. The images are aligned with respect to the bottom of the QW interfaces, as indicated by an arrow.

clearly visible. In both samples, the bottom interfaces to the interlayers (indicated by arrow in the images) appear horizontally flat and vertically atomically abrupt. This contrasts the top interfaces, which are diffuse. Regardless, the structural order at the QWs follows the wurtzite arrangement, without the nucleation of structural imperfections such as misfit dislocations as investigated by performing FFT filtering (not shown), with the exception of H5, which locally exhibits more pronounced structural distortions. Hence, it is 
(a) L-In (b) Binned to line

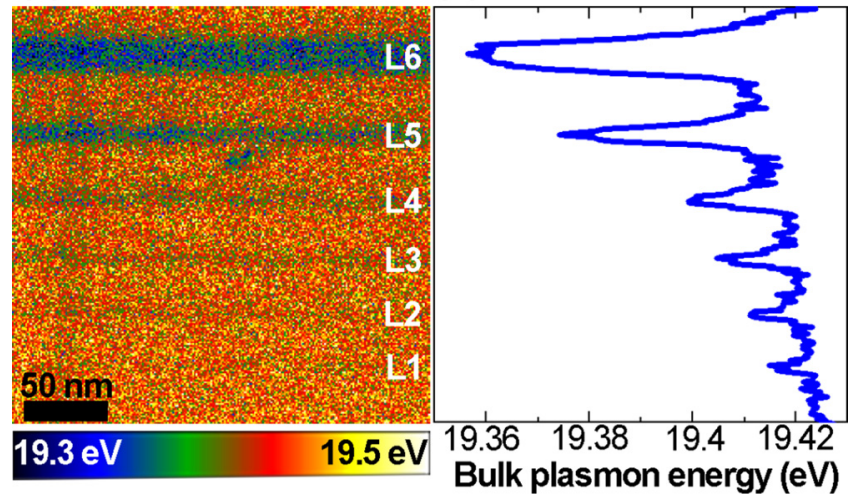

FIG. 4. (a) $\mathrm{E}_{\mathrm{p}}$ distribution map from the L-In QWs structure (note different energy scale to Fig. 5) (b) and binned to a line.

concluded that the wells grow perfectly pseudomorphic with respect to the $\mathrm{GaN}$ interlayers.

In order to acquire information regarding the In content and the In distribution of the QWs, VEELS mapping was performed on the structures where the results are shown in Figures 4 and 5 . The peak energy position of the bulk plasmon is mapped in Figures 4(a) and 5(a) (note the different energy scales between maps), where bright yellow contrast corresponds to the high bulk plasmon energy $\left(\mathrm{E}_{\mathrm{p}}\right)$ of $\mathrm{GaN}$ $(\sim 19.44 \mathrm{eV})$ and the darker (lower $\left.\mathrm{E}_{\mathrm{p}}\right)$ corresponds to an increasing incorporation of In in the structure, though InN $(\sim 15.51 \mathrm{eV})$ is not present. The variation in In composition was corroborated by simultaneous EDX maps (not shown) though these were not used due to a lower signal to noise ratio as compared to the VEELS maps.

It was previously found that $\mathrm{E}_{\mathrm{p}}$ follows a linear relation with composition in the similarly immiscible material $\mathrm{Al}_{\mathrm{x}} \mathrm{In}_{1-\mathrm{x}} \mathrm{N} .^{21}$ While this was not yet shown for InGaN, it was proposed that the relation holds for similar materials. Hence, the herein measured $\mathrm{E}_{\mathrm{p}}$ may potentially be used to derive the well compositions.

Figures 4 and 5 reveal that non-uniformities and local fluctuations of $\mathrm{E}_{\mathrm{p}}$, hence compositional variations, are present inside most QWs as well as in the interlayer between $\mathrm{H} 4$ and H5. Bright layers are attributed to In-deficient while darker correspond to In-rich regions. While the L-In sample (a)

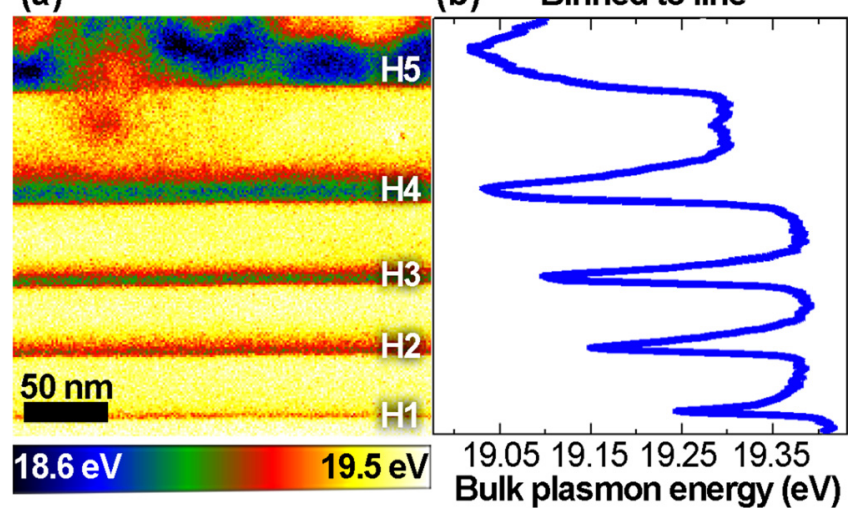

FIG. 5. (a) $E_{p}$ distribution map from the H-In QWs structure (note different energy scale to Fig. 4) and (b) binned to a line. contains significantly less In than the $\mathrm{H}$-In sample, the $\mathrm{E}_{\mathrm{p}}$ is not reproduced with the same energy range in Figure 4(a) as in 5(a). The $E_{p}$ map is naturally noisier for the L-In sample as the reduced In content influences $E_{p}$ less.

For an alternative view of the $\mathrm{E}_{\mathrm{p}}$ variations across the QWs, with improved signal-to-noise ratio, the laterally projected $E_{p}$ line profiles are shown in Figures 4(b) and 5(b). The L1 and L2 QWs are barely visible in the $\mathrm{E}_{\mathrm{p}}$ distribution maps; however, when averaged to a line, the increased signal to noise clearly resolves the presence of the QWs. In both investigated structures, there is an apparent and continuously increasing red shift of $E_{p}$ with increasing $Q W$ thickness suggesting an increased incorporation of In in the QWs, although $\mathrm{E}_{\mathrm{p}}$ shifts are of different order for H-In and L-In structures. Notably, the $E_{p}$ value does not blue shift back to the ideal $\mathrm{GaN}$ value $(19.44 \mathrm{eV})$ in the interlayers, which are separating the InGaN quantum wells. This indicates that some amount of In is present inside the interlayers causing this deviation (corroborated by EDX, not shown). In order to study the $E_{p}$ characteristics across the QWs with improved spatial accuracy, a set of the VEELS spectrum images were acquired from each $\mathrm{QW}$. The averaged $\mathrm{E}_{\mathrm{p}}$ line profiles across the QWs aligned with respect to the bottom of the QW interlayer interface (indicated by a red arrow) as identified from the simultaneously acquired HAADF image are shown in Figures 6 and 7 for the L-In and H-In structures.

The $\mathrm{E}_{\mathrm{p}}$ line profile shape across all individual QWs experiences similar distinct features and can be divided into two regions from bottom to top: (I) abrupt red shift, which for the thick wells levels out near the middle of the well, followed by (II) gradual blue shift resulting in asymmetric QW profiles. In the first region, going from GaN buffer/interlayers and into the InGaN QW layers, the sharp $\mathrm{E}_{\mathrm{p}}$ reduction correlates well with the HRSTEM observations and is connected with the increase in In content in the well upon introduction of TMIn into the growth zone. At this point, it

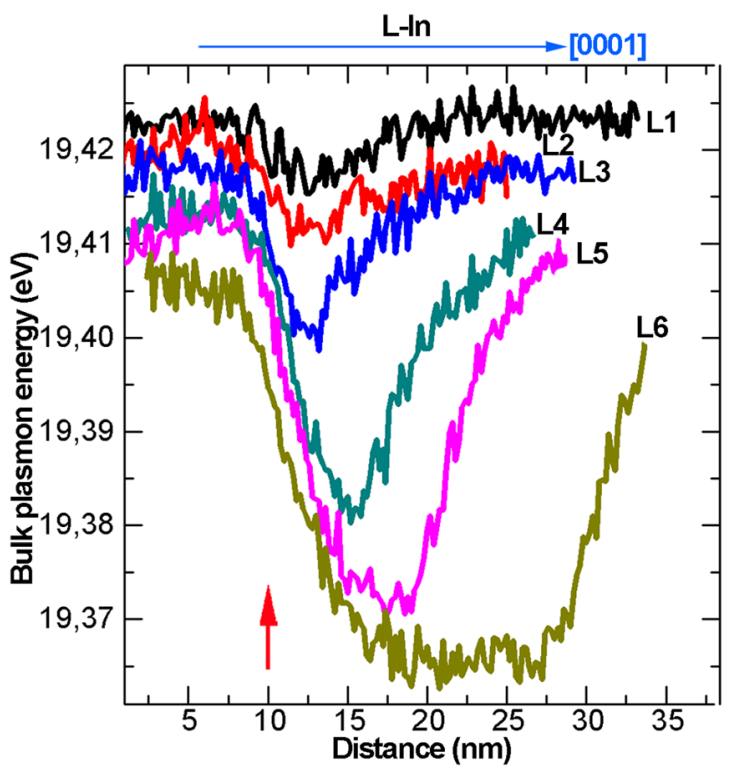

FIG. 6. Binned to line high spatial resolution $\mathrm{E}_{\mathrm{p}}$ distribution maps, showing $\mathrm{E}_{\mathrm{p}}$ dependence across each $\mathrm{QW}$ in L-In sample. Line profiles are aligned starting with the QW bottom, which is indicated by a red arrow. 


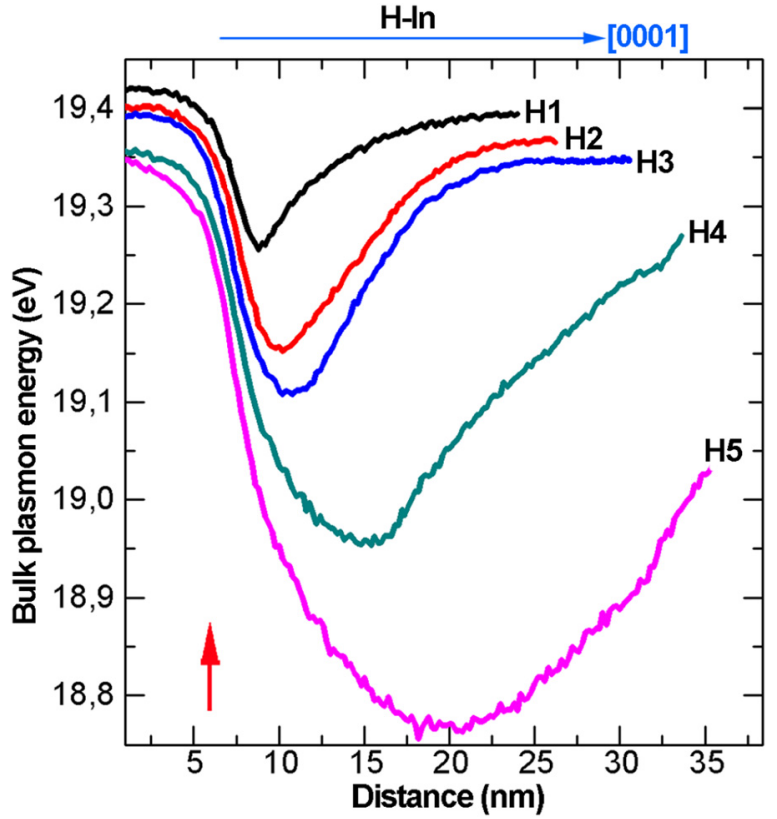

FIG. 7. Binned to line high spatial resolution $\mathrm{E}_{\mathrm{p}}$ distribution maps, showing $\mathrm{E}_{\mathrm{p}}$ dependence across each QW in H-In sample. Line profiles are aligned starting with the QW bottom, which is indicated by a red arrow.

should be noted that the spatial accuracy of the VEELS measurement is affected by beam broadening (due to scattering), beam geometry (convergent probe on sample of $\sim 50 \mathrm{~nm}$ thickness) and in particular delocalization of the bulk plasmon from both interlayer and QW as well as surface plasmons. ${ }^{16}$

The delocalization most likely gives the strongest contribution to the broadening of the $E_{p}$ line profiles, hence the integrated HAADF-STEM signal was compared with the $E_{p}$ line profiles across the QWs (not shown) to evaluate this contribution. It was concluded that the profiles are qualitatively similar and that the bulk plasmon delocalization effect contributes in a limited fashion to the shape of the $E_{p}$ profiles. The weak slope of the curves, just before the onset (indicated by the red arrow) of the QW is attributed to delocalization of the bulk plasmon, though the apparent consequence is minor. Hence, the slope of the well onset is therefore closely related to a compositional gradient inside the QWs. As the growth rate is constant through the sample, this can provide information on the rate of incorporation of the In at these conditions.

The influence of surface (sample to vacuum) plasmons on the measured spectra is neglected here. All measurements were performed in relatively thick regions (around 0.9 of the mean free path as evaluated by VEELS), with no detectable change to the spectra when moving towards thinner regions.

Additionally, confined structures introduce higher degree of complexity due to the presence of interface plasmons. Interface plasmon contribution was investigated by examining $\mathrm{GaN}$ spectra in different regions close to interface (not shown), which proved that no significant change of spectral features, and those findings agrees well with HAADF-STEM signal and $\mathrm{E}_{\mathrm{p}}$ line profiles comparison results discussed above.
It can be seen that the slope of the $E_{p}$ profile is identical for the first $2-3 \mathrm{~nm}$. This is likely due to identical growth and In incorporation rates. The middle of the well looks slightly different for all profiles and this will be addressed in conjunction with the discussion related to the $3 \mathrm{D}$ plots shown in Figure 8. As TMIn is removed from the flow, the In concentration is gradually reduced ( $E_{p}$ increases), which is seen in the second region of the profile. However, the rate at which the In concentration is reduced is significantly slower as compared to the increase of In into the well at the onset of the well growth. Since the gas flow speed in the MOCVD reactor is quite high, and the growth rate comparably slow, the observed tails cannot be explained by enduring TMIn alone. Hence, the tails are suggested to be a consequence of residual In, segregating on the surface of the sample before desorption or incorporation. ${ }^{22}$ As can be seen from the onset energy for each ticker $\mathrm{QW}, \mathrm{E}_{\mathrm{p}}$ is slightly reduced for each well even after $>30 \mathrm{~nm}$ of $\mathrm{GaN}$ interlayer growth, due to the increasing amount of residual In segregating on the surface with increasing well thickness. To estimate the maximum amount of incorporated In in each well, $E_{p}$ value was used. Assuming a linear dependence of $E_{p}$ with changing $\operatorname{In}_{\mathrm{x}} \mathrm{Ga}_{1-\mathrm{x}} \mathrm{N}$ composition from $\mathrm{GaN}\left(\mathrm{E}_{\mathrm{p}}=19.44 \mathrm{eV}\right)$ to $\operatorname{InN}\left(\mathrm{E}_{\mathrm{p}}=15.51 \mathrm{eV}\right)$, the maximum In incorporation in the respective QWs is summarized in Table $\mathrm{I}$. The lowest $\mathrm{E}_{\mathrm{p}}$ value at $18.75 \mathrm{eV}$ as observed in $\mathrm{H} 5$ is equivalent to an In incorporation of $\mathrm{x}=0.175$ and it should be noted that as low as $\mathrm{x}=0.0083$ was observed in L1. These compositional estimates are made considering a strain-free growth of the
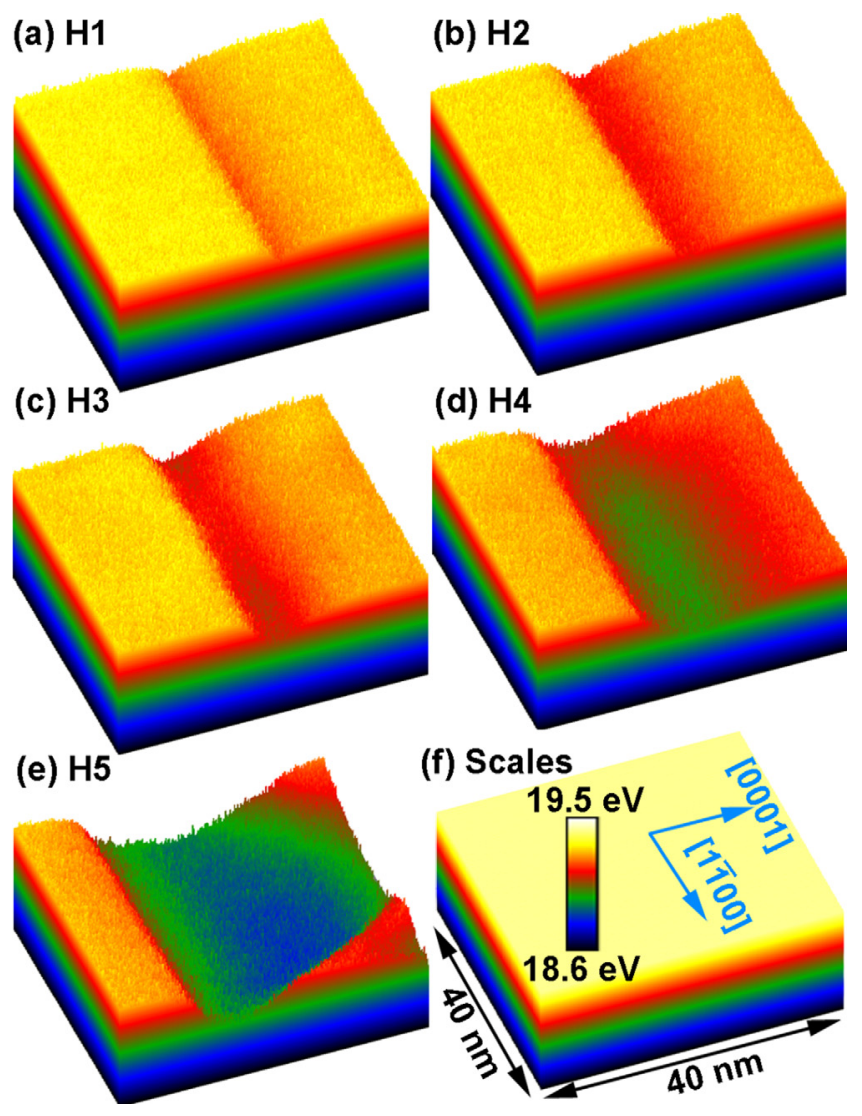

FIG. 8. High spatial resolution $\mathrm{E}_{\mathrm{p}}$ distribution maps of the H-In QWs starting from $\mathrm{H} 1$ (a) to $\mathrm{H} 5$ (e) and with scales and orientation indicated in (f). 
TABLE I. $\operatorname{In}_{\mathrm{x}} \mathrm{Ga}_{1-\mathrm{x}} \mathrm{N}$ QW characterization summary.

\begin{tabular}{lccc}
\hline \hline QW name & Average QW thickness, $\mathrm{nm}$ & $\mathrm{E}_{\mathrm{p}}, \mathrm{eV}$ & $\mathrm{x}$ \\
\hline $\mathrm{InN}$ & $\ldots$ & 15.51 & 1 \\
L1 & 1.9 & 19.415 & 0.0083 \\
L2 & 2.4 & 19.409 & 0.0091 \\
L3 & 3.5 & 19.398 & 0.0122 \\
L4 & 7.8 & 19.382 & 0.0163 \\
L5 & 10.8 & 19.371 & 0.0192 \\
L6 & 20.1 & 19.368 & 0.0214 \\
H1 & 5.4 & 19.263 & 0.0484 \\
H2 & 7.8 & 19.154 & 0.0757 \\
H3 & 8.4 & 19.12 & 0.0874 \\
H4 & 15.6 & 18.954 & 0.1253 \\
H5 & 25.4 & 18.765 & 0.1754 \\
GaN & $\ldots$ & 19.44 & 0 \\
\hline \hline
\end{tabular}

$\mathrm{In}_{\mathrm{x}} \mathrm{Ga}_{1-\mathrm{x}} \mathrm{N} \mathrm{QW}$, as strain has been concluded to contribute to a shift of $\mathrm{E}_{\mathrm{p}}{ }^{23}$ To grow strain-free $\operatorname{In}_{\mathrm{x}} \mathrm{Ga}_{1-\mathrm{x}} \mathrm{N}$ on $\mathrm{GaN}$ strain must first be relaxed by a number of misfit dislocations or other more notable structural defects. This is not the case in the investigated structures as a perfectly pseudomorphic growth was already concluded. Hence, the contents of the QW under investigation must be subject to compressive strain, hence $E_{p}$ is expected to experience a redshift, thus, the values in Table I are likely to be slightly underestimated. To accurately describe the strain of the QWs is, however, more difficult, as discussed below.

Three-dimensional images of the H-In QWs, for increasing well widths, are shown in Figures 8(a)-8(e). The dimensions represent area $(x, y)$ and $E_{p}(z)$ while the growth direction, and orientation are indicated in (f). The sharp onset of the well can be seen along with the slower recovery in all wells. Specifically, H3 and H4 in (c) and (d) exhibit interesting features inside the QWs. Looking at $\mathrm{H} 3$, there appears to be two elongated (in the plane of the well) features in the middle of the well, which are cut by the edges of the map and are seen in the color transition from "red" to "green." These features exhibit a lower $\mathrm{E}_{\mathrm{p}}$ and hence the local In concentration is higher as compared with the center of the map, between these features. Similarly, H4 exhibits a larger "green" feature in the middle of the well. At the edge of H4 (towards the spectator), the feature is cut, while at the opposing edge (away from the spectator), a narrow "red" bridge from top to bottom of the well can be identified, as well as the onset of another "green" feature, which is also cut by the edge of the map. The map of H5 also contains a large feature, though the growth of this layer is not continuous. Hence, any conclusions are difficult to extract from this map apart from the fact that H5 is not a perfect well since compositional "sidewalls" may be identified at the map boundaries.

In line with the colored map, the local lowering of $E_{p}$ indicates that these features exhibit a higher In concentration as compared to their environment, hence the observed features are suggested to act as QDs through a local reduction in bandgap and spatially limited distribution. It should be noted that no structural features (stacking faults/ dislocations) are present in these regions, hence no surface plasmons are present and able to presumably affect $E_{p}$ in this direction.

It is well known that for a given In composition of the InGaN layer, there is a critical thickness, at which a strained layer-by-layer growth of a $\mathrm{QW}$ is relaxed by assuming a Stranski-Krastanov-type growth. ${ }^{24}$ The QDs are then grown strain-free by relaxing strain in the ambient structure, e.g., plastically, by the introduction of misfit dislocations at the interface between wetting layer and dot. ${ }^{25,26}$ Here, a pseudomorphic growth was observed, hence no misfit dislocations are present to reduce the strain under the QDs. Typically, this is only possible by increasing the strain energy of the underlying substrate, ${ }^{27}$ resulting in a redshift of $E_{p}$ of the underlying substrate. Indeed, such an apparent shift of the well onset is subtly observed under the QDs (Figure 8(d)). This may, at a first glance, be identified as an undulating interface between interlayer and bottom of QW; however, the STEM images concluded this interface to be decidedly abrupt. Though it was proposed that the undulation of $E_{p}$ at the well onset is due to the increased delocalization of $E_{p}$, this could in part also be explained by strain adopted by the underlying interlayer.

As the TMIn supply is removed, the remaining liquid In on the surface acts as a source for continued growth of the InGaN QW. As the space between QDs is filled, the growth must occur laterally as well as vertically. Due to the continuously decreasing In source, the incorporated In concentration is continuously reduced as can be seen from the $E_{p}$ profiles.

Previously, it was observed that QDs formed by SK growth are dissolved after continued growth of $\mathrm{GaN} ;{ }^{28}$ however, here the QDs remain intact since the well continues to grow by drawing In from the In-rich surface, hence the QDs are buried inside the well. As the QWs are found to grow pseudomorphically, the In reduced InGaN in-between the QDs must be under strain. While the In reduced InGaN lattice has a smaller lattice as compared to the high In QDs, it serves to bridge the strain in the well as originally introduced by the QDs, also the amount of incorporated In in this layer is likely affected by the experienced strain. However, it is unlikely that the spacer layer absorbs the entire strain; some strain must be adopted by the QDs as well.

We find QDs in H-In QWs with average thickness $>8 \mathrm{~nm}$ (Table I), and where the In concentration corresponds to $\mathrm{x}>0.08$. In a previous study, it was inferred that QDs are formed and buried inside wells $(x=0.14)$ thicker than $5 \mathrm{~nm}$ (Ref. 29) although here we present a direct observation of quantum dots formed inside a quantum well. The results follow the critical thickness for transition to StranskiKrastanov-type growth.

The discussion on the weak luminescence quenching in the presence of high dislocation densities in InGaN has been active for decades. ${ }^{2,30}$ The common explanation is that charge carriers are somehow localized and thus nonradiative recombination at dislocations is significantly reduced. The presented mechanisms for localization may concern (1) only a few atoms, holes, or chains of such, ${ }^{31}$ (2) nanoscale non-random clustering of In atoms, i.e., by spinodal decomposition, ${ }^{32,33}$ or even (3) nanoscale fluctuations in the QW width, which could localize carriers. ${ }^{34}$ 
The quantum dots that have been presented herein are larger $(>20-30 \mathrm{~nm})$ than the In-rich clusters commonly observed after spinodal decomposition $(>2-5 \mathrm{~nm})$, but remain an organized (non-random) clustering of In atoms. Hence, these quantum dots are suggested to be an alternative, more stable source for carrier localization.

\section{CONCLUSIONS}

Quantum wells of different thickness and composition were investigated by monochromated STEM-VEELS at high spatial accuracy and energy resolution. The investigation shows how the InGaN/GaN QWs develop quantum dot like compositional fluctuations beyond a critical thickness of $>8 \mathrm{~nm}$ and corresponding In concentration $\mathrm{x}>0.08$. These dots are larger $(>30 \mathrm{~nm})$ than the regular In-rich clusters often found after spinodal decomposition $(\sim 2-5 \mathrm{~nm})$. The mechanism for quantum dot formation is suggested to originate from a coherent Stranski-Krastanov-type growth mode. The quantum dots are buried by overgrowth of Inreduced InGaN, where the In is incorporated from residual In segregating on the surface. Finally, the quantum dots are proposed to act as carrier localization centers and in part explain the origin for high luminescence in $\mathrm{InGaN}$ even in the presence of high dislocation densities.

\section{ACKNOWLEDGMENTS}

This work was supported by the Swedish Research Council (VR) through project and Linnaeus grants, the European Research Council (ERC), and the Swedish Foundation for Strategic research (SSF) through the Nano-N program. The authors also acknowledge the Knut and Alice Wallenberg Foundation for providing funding for the Linköping double-corrected $\operatorname{Titan}^{3}$ 60-300 kV electron microscope, and a Wallenberg Scholar Grant to L.H.

${ }^{1}$ H. Morkoc, Nitride Semiconductors and Devices (Springer-Verlag, Germany, 1999).

${ }^{2}$ S. Nakamura, Science 281, 956 (1998).

${ }^{3}$ B. Monemar, Phys. Rev. B 10, 676 (1974).

${ }^{4}$ J. Wu, J. App. Phys. 106, 011101 (2009).

${ }^{5}$ S. Nakamura, M. Senoh, N. Iwasa, and S. Nagahama, Jpn. J. Appl. Phys., Part 2 34, L797 (1995).
${ }^{6}$ A. Avramescu, T. Lermer, J. Muller, S. Tautz, D. Queren, S. Lutgen, and U. Strauss, Appl. Phys. Lett. 95, 071103 (2009).

${ }^{7}$ I. H. Ho and G. B. Stringfellow, Appl. Phys. Lett. 69, 2701 (1996).

${ }^{8}$ S. Y. Karpov, MRS Internet J. Nitride Semicond. Res. 3, 16 (1998).

${ }^{9}$ C. Kisielowski, Z. Liliental-Weber, and S. Nakamura, Jpn. J. Appl. Phys., Part 1 36, 6932 (1997).

${ }^{10}$ P. Ruterana, S. Kret, A. Vivet, G. Maciejewski, and P. Dluzewski, J. Appl. Phys. 91, 8979 (2002).

${ }^{11}$ D. Gerthsen, E. Hahn, B. Neubauer, A. Rosenauer, O. Schon, M. Heuken, and A. Rizzi, Phys. Status Solidi A 177, 145 (2000).

${ }^{12}$ U. Forsberg, A. Lundskog, A. Kakanakova-Georgieva, R. Ciechonski, and E. Janzen, Cryst. Growth Des. 311, 3007 (2009).

${ }^{13}$ A. Kakanakova-Georgieva, R. Ciechonski, U. Forsberg, A. Lundskog, and E. Janzen, Cryst. Growth Des. 9, 880 (2009).

${ }^{14}$ A. Gubbens, M. Barfels, C. Trevor, R. Twesten, P. Mooney, P. Thomas, N. Menon, B. Kraus, C. Mao, and B. McGinn, Ultramicroscopy 110, 962 (2010).

${ }^{15}$ M. Bosman and V. J. Keast, Ultramicroscopy 108, 837 (2008).

${ }^{16}$ R. E. Egerton, Micron 34, 127 (2003).

${ }^{17}$ A. Maigne and R. D. Twesten, J. Electron Microsc. 58, 99 (2009).

${ }^{18}$ M. Shiojiri, C. C. Chuo, J. T. Hsu, J. R. Yang, and H. Saijo, J. Appl. Phys. 99, 073505 (2006).

${ }^{19}$ X. H. Wu, C. R. Elsass, A. Abare, M. Mack, S. Keller, and P. M. Petroff, Appl. Phys. Lett. 72, 692 (1998).

${ }^{20}$ D. Holec, P. M. F. J. Costa, M. J. Kappers, and C. J. Humphreys, J. Cryst. Growth 303, 314 (2007).

${ }^{21}$ J. Palisaitis, C. L. Hsiao, M. Junaid, M. Xie, V. Darakchieva, J. F. Carlin, N. Grandjean, J. Birch, L. Hultman, and P. O. Å. Persson, Phys. Status Solidi (RRL) 5, 50 (2011).

${ }^{22}$ V. Potin, E. Hahn, A. Rosenauer, D. Gerthsen, B. Kuhn, F. Scholz, A. Dussaigne, B. Damilano, and N. Grandjean, J. Cryst. Growth 262, 145 (2004).

${ }^{23}$ J. Palisaitis, C. L. Hsiao, M. Junaid, J. Birch, L. Hultman, and P. O. A. Persson, Phys. Rev. B 84, 245301 (2011).

${ }^{24}$ S. Krastanov, Akad. Wiss. Wien. K1 146, 797 (1938).

${ }^{25}$ S. M. S. Pereira, K. P. O'Donnell, and E. J. C. Alves, Adv. Funct. Mater 17, 37 (2007).

${ }^{26}$ A. Pretorius, T. Schmidt, T. Aschenbrenner, T. Yamaguchi, C. Kubel, K. Muller, H. Dartsch, D. Hommel, J. Falta, and A. Rosenauer, Phys. Status Solidi B 248, 1822 (2011).

${ }^{27}$ S. Figge, C. Tessarek, T. Aschenbrenner, and D. Hommel, Phys. Status Solidi B 248, 1765 (2011).

${ }^{28}$ A. Pretorius, T. Yamaguchi, C. Kubel, R. Kroger, D. Hommel, and A. J. Rosenauer, J. Cryst. Growth 310, 748 (2008).

${ }^{29}$ Q. L. Zhang, F. Y. Meng, P. A. Crozier, N. Newman, and S. Mahajan, Acta Mater 59, 3759 (2011).

${ }^{30}$ R. A. Oliver, S. E. Bennett, T. Zhu, D. J. Beesley, M. J. Kappers, D. W. Saxey, A. Cerezo, and C. J. Humphreys, J. Phys. D 43, 354003 (2010).

${ }^{31}$ L. W. Wang, Phys. Rev. B 63, 245107 (2001).

${ }^{32}$ H. K. Cho, J. Y. Lee, N. Sharma, C. J. Humphreys, G. M. Yang, C. S. Kim, J. H. Song, and P. W. Yu, Appl. Phys. Lett. 79, 2594 (2001).

${ }^{33}$ Y.-T. Moon, D.-J. Kim, K.-M. Song, C.-J. Choi, S.-H. Han, T.-Y. Seong, and S.-J. Park, J. Appl. Phys. 89, 6514 (2001).

${ }^{34}$ C. J. Humphreys, Philos. Mag. 87, 1971 (2007). 